\title{
Isolated Interrupted Aortic Arch- A Complex Congenital heart disease
}

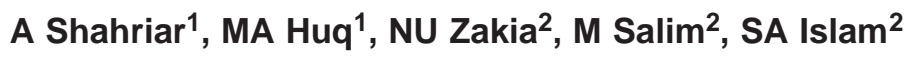 \\ ${ }^{1}$ Department of Pediatric Cardiology, NICVD, Dhaka. ${ }^{2}$ Department of Cardiology, NICVD, Dhaka
}

\author{
Keywords: \\ lnterrupted \\ aortic arch, \\ Complex \\ congenital heart \\ disease.
}

\begin{abstract}
:
Interrupted Aortic Arch (lAA) is the absence or discontinuation of a portion of the aortic arch. There are three types of Interrupted Aortic Arch,and they are classified according to the site of the interruption. lsolated IAA appears commonly in type B lAA, where the interruption occurs between the left carotid artery and the left subclavian artery.In such case there is no associated cardiac anomaly and the patient may not exhibit lethal form of presentation unless there is duct dependent circulation or ventricular septal defect. lsolated interrupted arch is relatively rare congenital anomaly and very few cases were reported in literature so far.
\end{abstract}

(Cardiovasc. j. 2011; 4(1): 102-104)

\section{Introduction:}

Interrupted aortic arch (IAA) is an uncommon complex congenital anomaly representing approximately $1 \%$ of congenital heart disease. The incidence is approximately 2 cases per 100,000 live births. More than $97 \%$ of the cases also have associated cardiac anomalies complicating their treatment. ${ }^{1}$ It occurs in association with a usually nonrestrictive ventricular septal defect and ductus arteriosus or, less commonly, with a large aorto pulmonary window or truncus arteriosus. ${ }^{2}$ Although most cases occur in normally connected great arteries, ${ }^{1}$ IAA can coexist with any ventriculoarterial ailgnment and also with single ventricle. ${ }^{1}$ IAA and complete common atrioventricular canal can be observed in the context of CHARGE association which is usually caused by mutations in CHDT on chromosome 8 q12.1. ${ }^{3}$ (coloboma, heart disease, atresia choanae, retarded growth and development and/or CNS anomalies, genital hypoplasia, and ear anomalies and/or deafness). Approximately $50 \%$ of IAA patients have DiGeorge syndrome and may exhibits facial dysmorphism. Isolated IAA cases without any other cardiac anamolies often go undetected into adolescence or early adult life, and then appear with a clinical picture similar to a postductal coarctation. ${ }^{1}$ In presence of severe cardiac anamaly symptoms $u$ sually occur early in the neonatal period and clinical deterioration is often rapid. ${ }^{4}$ Surgical reconstruction of the arch is now considered straightforward; hence, attention is increasingly focused on the preoperative identification and surgical management of the aortic valve and subaortic hypoplasia found in approximately half of cases. IAA is the first cardiovascular pattern formation anomaly to be demonstrated to have a genetic basis in both mouse and human.

\section{Case Report:}

9yrs old male afebrile child presented with severe dyspnoea and mild truncal oedema. On examination feeble peripheral pulse was felt in all four limbs. Oxygen saturation was $98 \%$ in all limbs without having any differential saturation. On CVS examination $\mathrm{S}_{1}$ was normal, S2 narrowly split, ESM found on left upper sternal area. The boy was stabilized in CCU with ionotrops and antifailure management. Echo colour Doppler showed subaortic membrane producing severe LVOT obstruction, interrupted aorticarch type $\mathrm{B}$, severe LV dysfunction, flow reversal in vertebral arteries, descending aorta and no PDA. In view of unusual diagnosis $\mathrm{CT}$ angiogram was done. $\mathrm{CT}$ angiogram shows isolated interrupted aortic arch, aberrant rt.subclavian artery, collaterals from descending aorta was supplied by vertebobasillar system. Patient went successful resection of subaortic membrane and repair of the interrupted aortic arch was done by Goretax gravt. He had a relatively smooth postoperative course and improvement of $\mathrm{LV}$ function by $40 \%$ during discharge. 


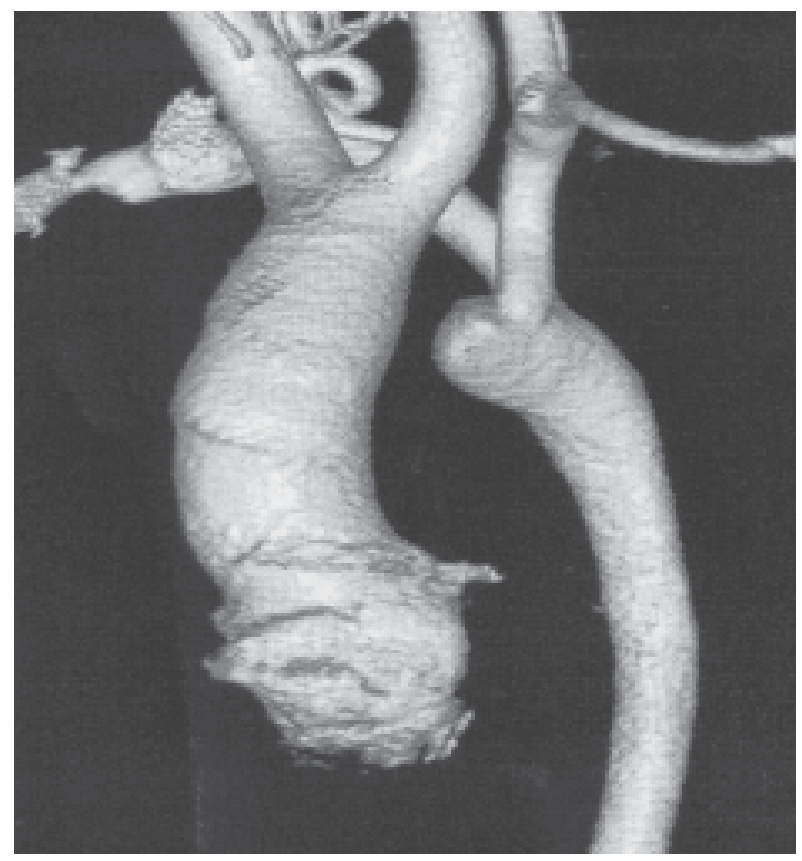

Fig.-1: CT angiogram shows interrupted aortic arch, aberrant rt subclavian artery and collaterals.

\section{Discussion:}

lnterrupted aortic arch has been classified in to 3 types $^{5}$ (A, B, and C) based on the site of aortic interruption. In type A interrupted left aortic arch, the arch interruption occurs distal to the origin of the left subclavian artery. In type B interrupted left aortic arch, the interruption occurs distal to the origin of the left common carotid artery.ln type $\mathrm{C}$ interrupted left aortic arch, the interruption occurs proximal to the origin of the left common carotid artery. In any of the 3 types, the right subclavian artery may arise normally or abnormally; the 2 most common abnormal sites are distal to the left subclavian artery (aberrant right subclavian artery) and from a right ductus arteriosus (isolated right subclavian artery). ${ }^{6}$ Type B interruptions account for about two thirds of cases, type A occur in about one third of cases, and type $\mathrm{C}$ are present in less than $1 \%$ of cases. Other than isolated IAA, most of the IAA are accompanied by various cardiac anomalies. Review study shows ventricular septal defect is present in $81 \%$, patent ductus arteriosus in $96 \%$, retro esophageal right subclavian artery in $12 \%$, truncus arteriosus in 10\%, atrial septal defect in $42 \%$, bicuspid aortic valve in $15 \%$, and various other infrequent defects run with IAA.
Type A IAA is formed by the abnormal regression of the left fourth arch segment late in development after the left subclavian artery has ascended to its normal position. Type B IAA results from involution of fourth arch and dorsal between arches four and six.Type C IAA seems to represent involution of the ventral portion of the left third arch and the left fourth arch. ${ }^{7}$

Recognizing IAA is difficult prior to reduction in the caliber of the ductus arteriosus. The hallmark there after is a mottled or grey appearance to the lower body, representing poor perfusion to that portion of the circulation located distal to the arch interruption. A difference in systolic blood pressure between the right arm and the lower extremities may or may not be present.Frequently, a lack of discrepancy in blood pressure is due to the profound reduction in cardiac performance. If the right subclavian artery is aberrant, no disparity occurs between the systolic blood pressure in the right arm and that in the lower extremities because the right subcravian origin is distal to the arch interruption. Although a difference in oxygen saturation between the right arm and the lower body may occur in cases without an aberrant right subclavian artery, this can be quite subtle in cases of high pulmonary blood flow. In normally connected great arteries, the oxygen saturation is higher in the right arm than in the lower body. In IAA with transposition of the great arteries, the reverse occurs.

Two-dimensional echocardiograpy is diagnostic for interrupted aortic arch. In addition, it can usually provide at least indirect evidence for the presence or absence of aberrant right subclavian artery. Occasionally, the presence of an isolated right subclavian artery can be detected. A suprasternal frontal sweep followed by left oblique and sagittal cuts is recommended. The most important contribution of 2-dimensional echocardiography to the preoperative characterization of patients with interrupted aortic arch is the display of the aortic outflow region. The presence of thymus can be ascertained as well Echocardiography also demonstrates the site of arch interruption, the size and anatomic type of the ventricular septal defect, the morphology of the aortic valve, and the anatomic severity of subaortic hypoplasia. Aortic valve and subaortic abnormalities are present in 
$50-80 \%$ of patients with interruptead aortic arch.

Cardiac catheterization or $\mathrm{CT}$ angiogram reveals the site of arch interruption, the size and anatomic type of ventricular septal defect, and the anatomic severity of subaortic hypoplasia. Cardiac catheterization also reveals whether the right subclavian artery is aberrant or not.

Evaluation of interrupted aortic arch (IAA) as an inpatient in an intensive care setting is advised. Intravenous prostaglandin E1 $(0.01 \mu \mathrm{gm} / \mathrm{kg})$ is indicated promptly to maintain patency of the ductus arteriosus in case of neonate with multiple cardiac anamaly. The need for an arterial line and assisted ventilation can be judged best from the initial ABG measurement as there may be associated metabolic acidosis.

The arch interruption itself is usually treated with side-to-side anastomosis, rather than with conduit interposition. If the subaortic region is of good size, the ventricular septal defect is usually closed with a patch at the same occasion. A recent study reported the successful use of a regional cerebral perfusion technique to correct interrupted aortic archl. ${ }^{10}$

Our patient didn't have any differential cyanosis as lower limbs are perfused with oxygenated blood from vertebrobasial system. 2nd heart sound was audible with physiological splitting and there is ESM on the upper sternal area due to LVOT obstruction. Total correction of IAA can be done in a single stage surgery with good result.

\section{References:}

1. Dische MR. Solitary interruption of the arch of the aorta: Clinicopathologic review of eight cases. Am J Cardiol 1975;35:271-277.

2. Gruber PJ, Epstein JA. Development gone awry: congenital heart disease. Circ Res 2004; 94(3): 273- 283.

3. Jongmans MC, Admiraal RJ, van der Donk KP, Vissers LE, Baas AF, Kapusta L. CHARGE syndrome: the phenotypic spectrum of mutations in the CHD7 gene. $J$ Med Genet 2006; 43: 306-314.

4. Van Praagh R. Interrupted aortic arch: Surgical treatment Am J Cardiol 1971; 27:200-210.

5. Celoria GC, Patton RB. Congenital absence of the aortic arch. Am Heart J 1959; 58:407-413.

6. Michael JR, Grady LH, Denton AC. Interrupted Aortic Arch: Brief Review and Summary of an Eighteen-Year Experienc. Texas Heart Inst $J$ 1984;1: 250-259.

7. Mulay AV,Watterson KG. Isolated right subclavian artery, interrupted aortic arch, and ventricular septal defect. Ann Thorac Surg 1997; 631:613 -615.

8. Apfel HD, Levenbraun J, Quaegebeur JM. Usefulness of preoperative echocardiograpy in predicting left ventricular outflow obstruction after primary repair of interrupted aortic arch with ventricular septal defect. Am J Cardiol 1998; 82.470- 473.

9. Cooley DA. Techniques in cardiac surgery. Philadelphia, WB Saunders,1984: 305-36.

10. Zhang H,Cheng P, Hou J, Li L, Liu H, Liu R, et al. Regional cerebral perfusion for surgical correction of neonatal aortic arch obstruction. Perfusion. Sep 16 2009; [Medline]. 